\title{
The comprehensive review of the neurovascular supply of the ankle joint: clinical implications
}

\author{
Chirapat Inchai ${ }^{1,2}$, Tanawat Vaseenon ${ }^{3}$, Pasuk Mahakkanukrauh ${ }^{2,4}$ \\ ${ }^{1} \mathrm{PhD}$ Degree Program in Anatomy, Department of Anatomy, Faculty of Medicine, Chiang Mai University, Chiang Mai, ${ }^{2}$ Department of Anatomy, \\ Faculty of Medicine, Chiang Mai University, Chiang Mai, ${ }^{3}$ Department of Orthopedics, Faculty of Medicine, Chiang Mai University, Chiang Mai, \\ ${ }^{4}$ Excellence in Osteology Research and Training Center (ORTC), Chiang Mai University, Chiang Mai, Thailand
}

\begin{abstract}
The rupture of ligament in the lateral part of ankle joint is a common injury and can lead to chronic ankle instability and lead to ankle osteoarthritis. Ankle arthroscopy is considered as a standard option to treat various ankle problems due to the need for only minimal incisions and fewer complications when compared to open surgery. However, there are complications associated with arthroscopic surgery e.g. damage to the anatomical structures around the portal placement areas. The present review provides anatomical knowledge of the superficial and deep neurovascular structures in the ankle region. These structures are important when ankle surgery is performed in order to avoid any intraoperative injury and prevent any complication following surgery.
\end{abstract}

Key words: Anatomy, Ankle, Arthroscopy, Nerve, Injury

Received February 19, 2020; Accepted March 13, 2020

\section{Introduction}

The ankle or talocrural joint is one of the most commonly injured joint compare to the other joint in human body while sprain or rupture of ankle ligaments are usually occurred in sport and in activities associated with daily living [1]. The lateral ligament complex in the lateral part of ankle joint is the most often damaged part of the ankle $[2,3]$. Approximately, $85 \%$ of injuries to the ankle are lateral ankle sprains [4]. Approximately $20 \%$ of acute rupture of ligament can develop into chronic instability of the ankle [5] which results from repetitive ankle sprains and incomplete healing of ligaments after injury $[6,7]$.

Nowadays, ankle arthroscopy is a minimal invasive technique, which is used to solve the problem of chronic lateral

\section{Corresponding author:}

Pasuk Mahakkanukrauh (iD

Department of Anatomy \& Excellence in Osteology Research and Training Center (ORTC), Chiang Mai University, Chiang Mai, Thailand

E-mail: pasuko34@gmail.com ankle instability when conservative management is unsuccessful. Since this technique requires minimal surgery and fewer complications a detailed and morphological structures of the ligament in the normal condition is necessary and needed to define for safety and effective in surgical treatment $[8,9]$. The complication rate in arthroscopic surgery for reconstruction the ligament in lateral part of ankle joint has been reported to be in the range of $9 \%$ to $17 \%$, and the most common complication from surgery is damage to the neurovascular structures. The superficial peroneal nerve (SPN) is one of the most commonly injured structures, about $3 \%$ to $8.5 \%$ of all arthroscopic complications [10-13]. It is not only the SPN that injured, however, as there are several anatomical structures which are also at risk of injury when treated using the arthroscopic approach or ankle surgery. The present review discusses the anatomical structures around the ankle joint especially in the lateral part which are important in arthroscopic surgery and these include the common peroneal, superficial peroneal, deep peroneal nerves and the dorsalis pedis artery. 


\section{Anatomy of the Neurovascular Supply to the Ankle Joint}

\section{The common fibular (peroneal) nerve}

The common fibular (peroneal) nerve or CPN is a small terminal branch from sciatic nerve, which is located on the lateral site of the leg and passes superficially to the neck of the fibula and separates into two terminal branches, the superficial peroneal or SPN and deep peroneal nerves or DPN. Damage to the CPN is most commonly found in all peripheral nerve injuries of the lower extremity. The CPN can be injured at various locations of the thigh and leg. Injury may be due to trauma or accidents which involve fracture of the femur and fibula especially in the fibular head region, bullet wounds, and direct injury. Traumatic causes may include accidents from motor vehicle wounds, fractures and postoperative entrapment. A research study studied the anatomical characteristics of the CPN, gross morphological study of the nerve and branching pattern, and surrounding structures i.e. the bones and the muscles in order to create a protocol for pre-operative and post-operative evaluation of patients undergoing surgical treatment [14]. A total of 20 lower legs of cadavers were dissected and used to carry out a study of the branching pattern of the CPN and its anatomical characteristic properties. The nerve divided in lower leg into 3 equal parts: the proximal, middle and distal, the dissection showed $76.5 \%$ of motor branches of the common peroneal nerve found in the proximal one third of the leg, and showed $19.5 \%$ of motor branches of the common peroneal nerve located in the middle part of leg, around 3.8\% showed the motor branches located in distal one third of leg. Hence, the researchers could investigate the safe zone for avoiding any injury to the $\mathrm{CPN}$ and its branches.

\section{The deep peroneal (fibular) nerve}

The deep peroneal (fibular) nerve or DPN originates from the CPN above the ankle joint. At the anterior part of the leg the DPN runs between the peroneus longus muscle and the neck of the fibula and provides the motor branches to innervate the muscles include the tibialis anterior (TA) muscle, the extensor digitorum longus (EDL) muscle, the extensor hallucis longus (EHL) muscle and the peroneus tertius muscle, which locate in the anterior compartment of leg [15]. After its passage into the anterior compartment, the DPN accompanies the anterior tibial artery, passing between the TA muscle and EDL muscle and then passes forwards to the
EDL in front of the interosseous membrane together with the anterior tibial artery and then descends with it to the ankle. In the ankle it divides there into lateral and medial terminal branches [14]. The DPN runs across the ankle joint and exits the compartment to supply the extensors digitorum and hallucis brevis and provide the sensory branches to innervate the skin of the foot [16]. The most common injury to the DPN is entrapment or compression when the nerve passes through the superior extensor retinaculum at the ankle joint level. This condition has been termed as anterior tarsal tunnel syndrome. There are many structures passing through the anterior tarsal tunnel i.e. deep peroneal nerve, dorsalis pedis artery, vein and the four tendons, which are the EHL, the TA, the EDL, and the PT tendons. Compression of the DPN is mostly by the tendon of the EHL and the extensor hallucis brevis.

\section{The superficial fibular (peroneal) nerve}

The superficial fibular (peroneal) nerve or SPN is one of the terminal branches of the CPN which is the nerve of the lateral compartment of the leg providing branches to innervate the muscles such as peroneus longus and peroneus brevis muscles. The SPN becomes a superficial nerve to innervate the skin on the distal part of the anterior surface of the leg and dorsal part of the foot. Takao et al. [17] studied the anatomy of the structures of the lower leg including the DPN and SPN to inform the placing the incision portal for ankle arthroscopy. A total of 51 ankles from 29 fresh cadavers which were aged between 51 and 95 years were used in the study. The study was conducted on gross dissection of the SPN and classified into 5 types. The results showed many different types of branching pattern of the SPN in the lower leg. At the ankle joint line, two branches of the SPN were found, in $32 \%$ of all specimens. Branches of the SPN were found in $11.8 \%$ cases, just lateral to the border of the tendon of peroneus tertius muscle while in $27.5 \%$ of all dissections, branches of SPN just medial to the border of peronius tertius muscle and tendon were found. This suggests that to avoid damage to the SPN and prevent complications the anterocentral portal is inappropriate for arthroscopy. Barrett et al. [18] performed a study into the lower leg to investigate the morphological variation of the SPN. A total of 75 specimens were dissected to improve further knowledge about the anatomical variations of the SPN in the middle one third of the lower leg. The results showed that the SPN was found in all compartments of the leg, most commonly in the lateral com- 
partment (72\% of all specimens). These findings suggested that the SPN could be injured from surgical procedures performed in the anterior and lateral parts of the leg and surgeons need to be aware of this to prevent complications. Darland et al. [19] studied the pattern of the branching of the SPN in fresh cadavers. A total of 50 ankle specimens were dissected to expose the SPN and classified following the Takao branching pattern classification [17]. The results showed various types of variation of the SPN branching pattern and location at the ankle joint and found a Takao type II was a typical type in this study, specifically two nerve branches at the level of the ankle joint. The results confirmed that the branching pattern of the SPN varied in many ways and the location of nerve at the level of the ankle joint in the zone of portal placement was therefore found to focus the need for extreme caution during surgical procedures of the ankle. Research study examined the anatomical variation of branching patterns of the DPN at the level of ankle joint [15]. A total of 17 cadaver specimens were dissected to observe and quantify the branching patterns of the DPN. The results showed that the DPN was located between the EHL muscle and TA muscles in the distal part of lower leg, and the DPN ran superficial to the anterior tibial artery. At approximately $12.5 \mathrm{~mm}$ proximal to the ankle joint line, the DPN crossed the tendon of the EHL and was located between the tendon of EHL and EDL. The DPN usually divided into two branches; the lateral the medial terminal branch at the levels of $12.4 \mathrm{~mm}$ distal to the mortise. Nayak and colleagues [20] conducted the studied of different patterns of nerve distribution on the dorsum of foot, 40 formalin fixed cadavers of 30 males and 10 females was dissected and classified the distribution pattern into 4 groups. The group I was the most common type (45\%), the DPN was supply the skin of the first interdigital space, the SN was supply the skin at the lateral side of 5th toe and the SPN was supply the remaining part of the dorsum of foot. Entrapment or compression of the SPN is an uncommon and rare cause of chronic pain in the lower leg $[21,22]$. The impingement of the SPN is commonly found as the SPN passes through the deep fascia of the leg into the anterior compartment [23]. It may be trapped as a result of anatomical variation including a thickness of fascial tunnel, a fascial defect and muscle herniation [24]. Injuries of the SPN can occur in sports activities or fracture dislocation of the upper tibia and fibula and are also at risk of direct injury from any procedure such as a total knee arthroplasty or TKA, ankle arthroscopy especially via the anterolateral portal [24].

\section{Arteries in the lateral and medial parts of ankle joint}

Pseudoaneurysms at the level of the ankle joint are rare and can develop following an ankle sprain, ankle fracture or as a complication after ankle arthroscopy [25]. There are case reports of pseudoaneurysms involving the anterior tibial artery occurring after ankle arthroscopic surgery [25, 26]. The dosalis pedis artery (DPA) is the continuation of the anterior tibial artery and passes through the anterior surface of the ankle joint. When ankle surgery is considered in order to solve an ankle problem, the DPA and its branches are some of the structures that can be injured when surgery is performed. Previous studies have demonstrated the variation of branching pattern of the DPA. Researchers performed a study on anatomical variation of the branching pattern of the DPA and found a wide variation in the location of arterial branches crossing the ankle joint [27]. Few other researchers studied the origin, branching pattern and direction of the anterior medial malleolar artery (AMMA), conducting the study on Japanese cadavers using computed tomography (CT) imaging [25]. The results showed variations in the origin of the AMMA at the level of the ankle joint line, below the ankle joint line and above the ankle joint line. The surgeon needs to be extremely aware of this during ankle arthroscopy surgery.

\section{Injury and etiology of the structure in the lateral part of ankle}

The position of the foot and ankle in plantar flexion, inversion and internal rotation of ankle joint are mechanisms of injury frequently found in damage to lateral ankle ligament as a result of complete or incomplete tear of anterior talofibular ligament (ATFL) that is the most thin and weakest ligament in the lateral part of ankle joint and calcaneofibular ligament (CFL) leading to chronic ankle instability [28, 29]. Approximately $75 \%$ of common ankle sprains involve a complete tearing of ligaments [30]. The ATFL tears in $80 \%$ patients, while the rupture both of ATFL and CFL occurs in the remaining 20\% [29-31]. A rupture of only ATFL and rupture both of ATFL and CFL, results in increased anterior displacement [32]. Conservative, nonsurgical treatment is considered as the primary treatment for most of the ankle ligament injuries. Although ankle stability was restored in most patients with nonsurgical treatment, in between $10 \%$ and $40 \%$ of cases conservative treatment was unsuccessful and patients developed chronic ankle joint instability with the presence of persistent pain, symptomatic, mechanical 
and functional instability and surgery was necessary to restore complete mobility.

\section{Current treatment of chronic ankle instability}

The primary management of chronic ankle instability for restoring stability of the ankle is conservative treatment [33, 34]. Secondary management is to prevent complications after injury, such as ankle joint instability which leads to ankle osteoarthritis. Conservative treatment includes the Rest, Ice, Compression, and Elevation techniques and provision of ankle support [35]. Surgical treatment is considered to solve these problems when conservative treatment is not successful, and the ligaments become lax [36, 37]. Recently, many surgical procedures were established to decrease the laxity of ligament. The reconstruction of ligaments can be divided into two techniques, specifically anatomical and non-anatomical reconstructions [38, 39]. Anatomical reconstructions yield good outcomes in the long term when compared to non-anatomical reconstruction [40-42].

\section{Minimal invasive techniques}

Arthroscopic reconstruction of ligaments is a minimal invasive surgical procedure. The outcomes of the technique has improved steadily over the past 20 years. Precise knowledge of the anatomy of the location and attachment areas of each ligament is necessary in order to perform anatomic reconstruction to address ankle instability problems successfully and also to have minimal complications after surgery. A search of extant literature revealed many studies which considered the ligament in the lateral part of ankle joint in terms of gross variation of each ligament, the appropriate tendon for all autografts and allografts and the success rate of reconstructive techniques for surgery. Few studies focus on the footprint to provide a basic knowledge of the attachment area of the ligament in the lateral part of ankle. Basic knowledge of the anatomical structures in the ankle region are essential for orthopaedic surgeons in choosing the site for the minimal invasive technique giving fewer complications for their patients [43-46].

\section{Anatomical study related to arthroscopic surgery}

There are many important anatomical structures at the ankle joint level. When performing surgical procedures such as ankle arthroscopic surgery for repair or reconstruction of the lateral ligament, a clear understanding of the surrounding structures is important to help the surgeon to avoid complications. There has been a lack of thorough anatomic study defining the safe zones and structures at risk from arthroscopic repair. There are many previous studies that have focused on the anatomical structures related to the surgical treatment including the neurovascular structures. According to researchers who studied the anatomical structures surrounding the lateral part of ankle joint, both superficial and deep structures are at risk after arthroscopic surgery to solve lateral ankle ligament problems [47]. The results showed the high risk of entrapment of the tendons in the anterior compartment of leg and entrapment of the SPN especially in which intermediate branch occurred in two ankle specimens from five ankle specimens. Researchers conducted a study on 30 ankles of 15 fresh cadavers for the safety zone for the surgical approach for arthroscopy by placing 2 portals to the subtalar arthrodesis [48]. They suggested that the two lateral portals approach may effective and safe for ankle arthroscopic arthrodesis of the posterior subtalar joint. Other researchers conducted an anatomical study on 15 lower legs from fresh cadavers to determine the relationship between the anatomical structures in the lateral part of ankle that may be impacted in arthroscopic repair [49]. Awareness needs to be raised about the variation in origin of the ATFL to provide a safety area to better approach for ATFL repair through minimal invasive surgery. The results showed the greatest variation in the location of the SPN and demonstrated that the mean distance from the SPN to the ATFL was $25 \pm 6 \mathrm{~mm}$. Injury to the SPN is an important complication which is found in arthroscopic ATFL repair, suggesting that approximately $22 \mathrm{~mm}$ from the lateral malleolus, there was a high risk of damage to the SPN in the anterior direction of the ankle.

\section{Conclusion}

Ankle arthroscopy is frequently used to solve chronic ankle instability, which is a minimal invasive technique that can reduce complications after surgery. There are various anatomical structures that may be damaged during surgery. In this review, we highlighted the anatomical basis of neurovascular structures in the lateral part of ankle joint related to the clinical implications specific to the arthroscopic procedure. 


\section{ORCID}

Chirapat Inchai: https://orcid.org/0000-0002-0524-582X

Tanawat Vaseenon:

https://orcid.org/0000-0003-2903-7248

Pasuk Mahakkanukrauh:

https://orcid.org/0000-0003-0611-7552

\section{Author Contributions}

Conceptualization: CI, PM. Data acquisition: CI. Data analysis or interpretation: CI, TV. Drafting of the manuscript: TV. Critical revision of the manuscript: PM. Approval of the final version of the manuscript: all authors.

\section{Conflicts of Interest}

No potential conflict of interest relevant to this article was reported.

\section{Acknowledgements}

We would like to thank the Excellence in Osteology Research and Training Center (ORTC) with partially supported by Chiang Mai University.

\section{References}

1. Balduini FC, Tetzlaff J. Historical perspectives on injuries of the ligaments of the ankle. Clin Sports Med 1982;1:3-12.

2. Glasgow M, Jackson A, Jamieson AM. Instability of the ankle after injury to the lateral ligament. J Bone Joint Surg Br 1980;62-B:196-200.

3. Gutierrez GM, Kaminski TW, Douex AT. Neuromuscular control and ankle instability. PM R 2009;1:359-65.

4. Garrick JG. The frequency of injury, mechanism of injury, and epidemiology of ankle sprains. Am J Sports Med 1977;5:241-2.

5. Watt T, Hariharan AR, Brzezinski DW, Caird MS, Zeller JL. Branching patterns and localization of the common fibular (peroneal) nerve: an anatomical basis for planning safe surgical approaches. Surg Radiol Anat 2014;36:821-8.

6. Karlsson J, Lansinger O. Lateral instability of the ankle joint. Clin Orthop Relat Res 1992;(276):253-61.

7. Chan KW, Ding BC, Mroczek KJ. Acute and chronic lateral ankle instability in the athlete. Bull NYU Hosp Jt Dis 2011;69:1726.

8. Guillo S, Bauer T, Lee JW, Takao M, Kong SW, Stone JW, Mangone PG, Molloy A, Perera A, Pearce CJ, Michels F, Tourné Y, Ghorbani A, Calder J. Consensus in chronic ankle instability: aetiology, assessment, surgical indications and place for arthroscopy. Orthop Traumatol Surg Res 2013;99(8 Suppl):S411-9.

9. Guillo S, Cordier G, Sonnery-Cottet B, Bauer T. Anatomical reconstruction of the anterior talofibular and calcaneofibular ligaments with an all-arthroscopic surgical technique. Orthop Traumatol Surg Res 2014;100(8 Suppl):S413-7.

10. Amendola A, Petrik J, Webster-Bogaert S. Ankle arthroscopy: outcome in 79 consecutive patients. Arthroscopy 1996;12:56573.

11. Bonnin M, Bouysset M. Arthroscopy of the ankle: analysis of results and indications on a series of 75 cases. Foot Ankle Int 1999;20:744-51.

12. Deng DF, Hamilton GA, Lee M, Rush S, Ford LA, Patel S. Complications associated with foot and ankle arthroscopy. J Foot Ankle Surg 2012;51:281-4.

13. Ferkel RD, Small HN, Gittins JE. Complications in foot and ankle arthroscopy. Clin Orthop Relat Res 2001;(391):89-104.

14. Reebye O. Anatomical and clinical study of the common fibular nerve. Part 1: anatomical study. Surg Radiol Anat 2004;26:365-70

15. Lawrence SJ, Botte MJ. The deep peroneal nerve in the foot and ankle: an anatomic study. Foot Ankle Int 1995;16:724-8.

16. Moore KL, Dalley AF, Agur AMR. Clinically oriented anatomy. 7th ed. Philadelphia: Wolters Kluwer/Lippincott Williams \& Wilkins, 2014

17. Takao M, Uchio Y, Shu N, Ochi M. Anatomic bases of ankle arthroscopy: study of superficial and deep peroneal nerves around anterolateral and anterocentral approach. Surg Radiol Anat 1998;20:317-20.

18. Barrett SL, Dellon AL, Rosson GD, Walters L. Superficial peroneal nerve (superficial fibularis nerve): the clinical implications of anatomic variability. J Foot Ankle Surg 2006;45:174-6.

19. Darland AM, Kadakia AR, Zeller JL. Branching patterns of the superficial peroneal nerve: implications for ankle arthroscopy and for anterolateral surgical approaches to the ankle. J Foot Ankle Surg 2015;54:332-7.

20. Nayak VS, Bhat N, Nayak SS, Sumalatha S. Anatomical variations in the cutaneous innervation on the dorsum of the foot. Anat Cell Biol 2019;52:34-7.

21. Rosson GD, Dellon AL. Superficial peroneal nerve anatomic variability changes surgical technique. Clin Orthop Relat Res 2005;438:248-52.

22. Styf J, Morberg P. The superficial peroneal tunnel syndrome. Results of treatment by decompression. J Bone Joint Surg Br 1997;79:801-3.

23. Tzika M, Paraskevas G, Natsis K. Entrapment of the superficial peroneal nerve: an anatomical insight. J Am Podiatr Med Assoc 2015;105:150-9.

24. Johnston EC, Howell SJ. Tension neuropathy of the superficial peroneal nerve: associated conditions and results of release. Foot Ankle Int 1999;20:576-82.

25. Tonogai I, Hayashi F, Tsuruo Y, Sairyo K. Examination of the origin and branching direction of the anterior medial malleolar artery from the anterior tibial artery: a cadaveric study. Foot 
Ankle Spec 2019;12:167-71.

26. Yu JL, Ho E, Wines AP. Pseudoaneurysms around the foot and ankle: case report and literature review. Foot Ankle Surg 2013;19:194-8.

27. Parikh S, Dawe E, Lee C, Whitehead-Clarke T, Smith C, Bendall S. A cadaveric study showing the anatomical variations in the branches of the dorsalis pedis artery at the level of the ankle joint and its clinical implication in ankle arthroscopy. Ann R Coll Surg Engl 2017;99:286-8.

28. Kovaleski JE, Hollis J, Heitman RJ, Gurchiek LR, Pearsall AW 4th. Assessment of ankle-subtalar-joint-complex laxity using an instrumented ankle arthrometer: an experimental cadaveric investigation. J Athl Train 2002;37:467-74.

29. Hubbard TJ, Wikstrom EA. Ankle sprain: pathophysiology, predisposing factors, and management strategies. Open Access J Sports Med 2010;1:115-22.

30. Brostroem L. Sprained ankles. I. Anatomic lesions in recent sprains. Acta Chir Scand 1964;128:483-95.

31. Neuschwander TB, Indresano AA, Hughes TH, Smith BW. Footprint of the lateral ligament complex of the ankle. Foot Ankle Int 2013;34:582-6.

32. Kovaleski JE, Heitman RJ, Gurchiek LR, Hollis JM, Liu W, Pearsall AW 4th. Joint stability characteristics of the ankle complex after lateral ligamentous injury, part I: a laboratory comparison using arthrometric measurement. J Athl Train 2014;49:192-7.

33. Ajis A, Maffulli N. Conservative management of chronic ankle instability. Foot Ankle Clin 2006;11:531-7.

34. Giannini S, Ruffilli A, Pagliazzi G, Mazzotti A, Evangelisti G, Buda R, Faldini C. Treatment algorithm for chronic lateral ankle instability. Muscles Ligaments Tendons J 2014;4:455-60.

35. Safran MR, Zachazewski JE, Benedetti RS, Bartolozzi AR 3rd, Mandelbaum R. Lateral ankle sprains: a comprehensive review part 2: treatment and rehabilitation with an emphasis on the athlete. Med Sci Sports Exerc 1999;31(7 Suppl):S438-47.

36. Karlsson J, Lansinger O. Chronic lateral instability of the ankle in athletes. Sports Med 1993;16:355-65.
37. Trevino SG, Davis P, Hecht PJ. Management of acute and chronic lateral ligament injuries of the ankle. Orthop Clin North Am 1994;25:1-16.

38. Al-Mohrej OA, Al-Kenani NS. Chronic ankle instability: current perspectives. Avicenna J Med 2016;6:103-8.

39. Becker HP, Rosenbaum D. [Chronic recurrent ligament instability on the lateral ankle]. Orthopade 1999;28:483-92. German.

40. Coughlin MJ, Schenck RC Jr, Grebing BR, Treme G. Comprehensive reconstruction of the lateral ankle for chronic instability using a free gracilis graft. Foot Ankle Int 2004;25:231-41.

41. Coughlin MJ, Schenck RC Jr. Lateral ankle reconstruction. 2001;22:256-8.

42. Wang B, Xu XY. Minimally invasive reconstruction of lateral ligaments of the ankle using semitendinosus autograft. Foot Ankle Int 2013;34:711-5.

43. Baumhauer JF, O'Brien T. Surgical considerations in the treatment of ankle instability. J Athl Train 2002;37:458-62.

44. Brodsky AR, O'Malley MJ, Bohne WH, Deland JA, Kennedy JG. An analysis of outcome measures following the BroströmGould procedure for chronic lateral ankle instability. Foot Ankle Int 2005;26:816-9.

45. Ferkel RD, Chams RN. Chronic lateral instability: arthroscopic findings and long-term results. Foot Ankle Int 2007;28:24-31.

46. Gould N, Seligson D, Gassman J. Early and late repair of lateral ligament of the ankle. Foot Ankle 1980;1:84-9.

47. Drakos M, Behrens SB, Mulcahey MK, Paller D, Hoffman E, DiGiovanni CW. Proximity of arthroscopic ankle stabilization procedures to surrounding structures: an anatomic study. Arthroscopy 2013;29:1089-94.

48. Lintz F, Guillard C, Colin F, Marchand JB, Brilhault J. Safety and efficiency of a 2-portal lateral approach to arthroscopic subtalar arthrodesis: a cadaveric study. Arthroscopy 2013;29:1217-23.

49. Jorge JT, Gomes TM, Oliva XM. An anatomical study about the arthroscopic repair of the lateral ligament of the ankle. Foot Ankle Surg 2018;24:143-8. 